\title{
Review: family intervention added to standard treatment for schizophrenia reduces relapse
}

\author{
Barbato A, D'Avanzo B. Family interventions in schizophrenia and related disorders: a critical review of clinical trials. \\ Acta Psychiatr Scand 2000 Aug;102:81-97.
}

\author{
QUESTIONS: In patients with schizophrenia, does family intervention increase the \\ efficacy of standard treatment, and what are the characteristics of successful \\ intervention?
}

\section{Data sources}

Studies were identified by searching Medline (1976-98), scanning reviews, and handsearching journal titles (1976-97) listed in Evidence-Based Mental Health.

\section{Study selection}

English language studies were selected if they were clinical trials of psychosocial treatment aimed at families of patients with schizophrenia and related disorders, used a standardised diagnostic system, were randomised controlled trials (RCTs) that compared family intervention with a control group or alternative family interventions, and systematically described and measured outcomes.

\section{Data extraction}

Data were extracted on number of patients in treatment groups, type of family intervention, patient characteristics, setting, outcome measures, and results.

\section{Main results}

25 studies (1744 patients) met the selection criteria. 15 studies compared relapse rates, which were defined in different ways across studies. Statistically significant lower relapse rates were reported for the intervention group than for the control group in 7 of 10 studies at 6-12 months. The median relapse rates across studies was $18 \%$ and $44 \%$ at 1 year and $33 \%$ and $64 \%$ at 2 years for the intervention and control groups, respectively. 1 study published in 1997 reported higher relapse rates for the intervention group than for the control group at 6-12 months ( $42 \%$ v 25\%). 6 studies compared changes in level of pathology in intervention and control groups. 1 study showed higher remission rates for the intervention group than for the control group (56\% v $22 \%$ at 9 mo and $67 \%$ v $17 \%$ at 2 y); 2 studies showed moderate treatment effect sizes of 0.34 and 0.38 on global psychopathology. Data from the other 3 studies were not adequate for drawing conclusions. 2 of 9 studies assessing social functioning outcome showed a moderate to substantial treatment effect size for family intervention ( 0.54 [estimated in non-relapsed patients only] and 1.43 [in 1 of 2 measures used]); 2 studies reported a small treatment effect size, 2 studies reported no statistically significant differences, and 3 studies had unclear or incomplete data. 14 studies examined the effect of family intervention on familyrelated variables; various measures were used, which limits the strength and comparability of findings. Most studies assessing psychoeducational and/or behavioural approaches had positive effects, whereas studies assessing other models showed negative results or had substantial methodological weaknesses. Studies in which the intervention was aimed at relatives without the patients present consistently showed negative results. Similarly, studies in which family intervention was provided in the short term $(\leqslant 10$ sessions over $<6$ mo) did not show long lasting results.

\section{Conclusions}

In patients with schizophrenia, the addition of family intervention to standard treatment reduces the risk of clinical relapse. Insufficient evidence exists on the effect of family intervention on mental state and social functioning or on family related variables. Successful intervention characteristics are the inclusion of the patient in treatment, long duration, and the use of information and education about the illness.

\section{COMMENTARY}

Evidence is increasing for the efficacy of psychosocial interventions in schizophrenia, and some patients (and families) prefer them over medications. ${ }^{1}$ There can, however, be difficulties with implementation in everyday clinical practice where resources are scarce, trained staff may not be available or may not have the time to see many patients, and providing such interventions over many years is different from being part of a team doing a randomised controlled trial. Indeed, variations in precisely what is given, by whom, and where and when may account for some of the inconsistencies in these studies. Many of the approaches combine overlapping elements, and if we knew what worked for which patients, it might be easier to provide a service.

Barbato and D'Avanzo in their review of family interventions replicate and extend an earlier version of an existing Cochrane review. ${ }^{2}$ They show that family interventions reduce relapse rates but also highlight several recent studies that have failed to find this. Many reasons are possible for this finding, but the most likely is that more recent studies are better controlled for non-specific therapeutic elements. The other possibilities worth mentioning are that earlier studies may have included more families with "high expressed emotion" or that patients just spend less time with their families than they used to. They also show that at least some of the effect of family intervention is to increase compliance with medication.

Barbato and D'Avanzo suggest that family interventions should provide information about the illness, include the patient, and be long term to have long term benefits. The latter result could reflect an important dose response effect. Alternatively, it may mean that non-specific therapeutic elements such as long term support are as important as anything else. The latter interpretation is compatible with what families actually say is beneficial about these approaches. ${ }^{3}$

The review by Merinder is arguably a more important contribution because it focuses on one element of many psychosocial interventionspsychoeducation, although I don't see why it can't be called "education." Information certainly appears to increase knowledge, but it is less clear that it changes attitudes to the illness or its treatment, improves compliance, or reduces relapse. The Cochrane review and meta-analysis of 10 of the RCTs included here, however, finds an overall effect on relapse. ${ }^{4}$ There is also one rather curious finding in the Merinder review, which may have substantial clinical relevance. continued on next page 


\title{
Review: patient education is effective for improving patient knowledge of schizophrenia
}

Merinder LB. Patient education in schizophrenia: a review. Acta Psychiatr Scand 2000 Aug;102:98-106.

\author{
QUESTION: In patients with schizophrenia, does patient education improve knowledge, \\ compliance, relapse, symptoms, social function, insight, and satisfaction?
}

\section{Data sources}

Studies were identified by searching Medline (1966-97), EMBASE/Excerpta Medica (1987-97), PsycLIT (197496), and CINAHL (1982-97) and by scanning reference lists of relevant articles.

\section{Study selection}

Studies were selected if they were randomised controlled trials (RCTs) of patient education in patients with schizophrenia (category A). Supplementary data were taken from naturalistic studies of patient education in patients with schizophrenia (category B) and RCTs of patient education in mixed samples (category C). Studies were excluded if they used complex interventions involving mainly social skills training and psychotherapy, or family intervention with educational programmes.

\section{Data extraction}

Data were extracted on patient characteristics, type of intervention, number of sessions, length of follow up, results, and study methods.

\section{Main results}

7 RCTs (category A), 4 naturalistic studies (category B), and 8 RCTs (category C) met the selection criteria. The 7 category A RCTs had sample sizes of 20-236 patients (mean 82 patients). Follow up ranged from 3 weeks to 2 years. 3 RCTs included an element of training that ranged from a 1 hour counselling intervention on how to tailor the prescribed regimen to a medication module for 3 hours weekly for 2-3 months. 1 RCT had a brief training element on relapse prevention. 1 RCT compared behavioural training with didactic methods. The other 2 RCTs used only didactic methods (1 hour to 25 hours weekly for $3 \mathrm{wks}$ ). The 8 category C RCTs had sample sizes of 28-418 patients (mean 95 patients). Follow up ranged from 2 weeks to 6 months. In 5 RCTs, the diagnoses were not reported; in 2 RCTs, the proportions of patients with schizophrenia were $42 \%$ and $65 \%$. 1 RCT reported that the diagnoses were "mainly" schizophrenia. 6 RCTs used group information together with discussion of medication issues (1 RCT), reinforcement (1 RCT), role play (1 RCT), and discussion on tardive dyskinesia (1 RCT). 1 RCT compared an information sheet only with an information sheet and verbal explanation, and 1 RCT used individual information and problem identification combined with compliance planning and problem solving. Most studies showed an improvement in knowledge (5 of 6 RCTs in category A and 5 of 6 RCTs in category C). Results for other outcomes were mixed: a treatment benefit was shown for compliance in 2 of 5 RCTs (category A) and 3 of 5 RCTs (category C); for relapse in 1 of 3 RCTs (category A) and 1 of 3 RCTs (category C); for symptoms in 2 of 5 RCTs (category A) and 1 of 1 RCT (category C); for social function in 2 of 4 RCTs (category A); for insight in 1 of 2 RCTs (category A); for quality of life in 1 of 1 RCT (category $\mathrm{A})$; and for satisfaction in 1 of 1 RCT (category A) and 0 of 1 RCT (category C).

\section{Conclusions}

In patients with schizophrenia, patient education is effective for improving knowledge. Results for compliance, relapse, symptoms, social function, insight, quality of life, or satisfaction are not conclusive.
Source of funding: no external funding.

For correspondence: Dr L B Merinder Department of Psychiatric Demography, Institute for Basic Psychiatric Research, Psychiatric Hospital in Aarhus, University Hospital, Skovagervej 2,

DK-8240 Risskor, Denmark. Fax +4577 892899 .

\section{COMMENTARY — continued from previous page}

It appears that short ( $<10$ sessions) and long interventions have no differential effect on improving knowledge or compliance. This could mean that only a certain amount of information can be given usefully to patients. It is also possible that family intervention effects result from more than just education.

Where does all this leave the practising clinician? The best available evidence shows that various psychosocial interventions can be beneficial in schizophrenia, but that the common elements of information about the illness and long term support may be as good as anything else. Patients clearly need to be involved, and it may only be necessary to involve their family if they are particularly critical or hostile (provided, of course, the patients have contact with them and everyone is amenable to this approach). A rolling group, with intermittent educational sessions, may be all that is required. It should be borne in mind that any effective intervention can have adverse effects and information could conceivably reduce morale and increase hopelessness. Finally, perhaps the greatest service one could give to one's patients and the global community of patients and clinicians would be to routinely audit the implementation of an approach, ask the patients and relatives to indicate what they find useful, and publish the results.

Stephen M Lawrie, MRCPsych, MPhil Royal Edinburgh Hospital Edinburgh, UK

1 Lawrie SM. Schizophrenia. Clin Evidence 2000;3:475-85.

2 Pharoah FM, Mari JJ, Streiner D. Family intervention for schizophrenia. Cochrane Database Syst Rev 2000;(4):CD000088.

3 Budd RJ, Hughes IC. What do relatives of people with schizophrenia find helpful about family intervention? Schizophr Bull 1997;23:341-7.

4 Pekkala E, Merinder L. Psychoeducation for schizophrenia. Cochrane Database Syst Rev 2000;(4):CD002831. 